\title{
H.264/AVC Video Compressed Traces: Multifractal and Fractal Analysis
}

\author{
Irini Reljin, ${ }^{1}$ Andreja Samčović, ${ }^{2}$ and Branimir Reljin ${ }^{1}$ \\ ${ }^{1}$ Faculty of Electrical Engineering, University of Belgrade, 11000 Belgrade, Serbia and Montenegro \\ ${ }^{2}$ Faculty of Traffic and Transport Engineering, University of Belgrade, 11000 Belgrade, Serbia and Montenegro
}

Received 1 August 2005; Revised 1 January 2006; Accepted 30 April 2006

Publicly available long video traces encoded according to H.264/AVC were analyzed from the fractal and multifractal points of view. It was shown that such video traces, as compressed videos (H.261, H.263, and MPEG-4 Version 2) exhibit inherent longrange dependency, that is, fractal, property. Moreover they have high bit rate variability, particularly at higher compression ratios. Such signals may be better characterized by multifractal (MF) analysis, since this approach describes both local and global features of the process. From multifractal spectra of the frame size video traces it was shown that higher compression ratio produces broader and less regular MF spectra, indicating to higher MF nature and the existence of additive components in video traces. Considering individual frames (I, P, and B) and their MF spectra one can approve additive nature of compressed video and the particular influence of these frames to a whole MF spectrum. Since compressed video occupies a main part of transmission bandwidth, results obtained from MF analysis of compressed video may contribute to more accurate modeling of modern teletraffic. Moreover, by appropriate choice of the method for estimating MF quantities, an inverse MF analysis is possible, that means, from a once derived MF spectrum of observed signal it is possible to recognize and extract parts of the signal which are characterized by particular values of multifractal parameters. Intensive simulations and results obtained confirm the applicability and efficiency of MF analysis of compressed video.

Copyright (C) 2006 Hindawi Publishing Corporation. All rights reserved.

\section{INTRODUCTION}

Video data is main and most critical part of modern multimedia communications due to its huge amount of data. For the transport over networks, video is typically compressed (or, encoded) to reduce the bandwidth requirements. The standardization activities in the field of video compression are in focus of two professional bodies: the ITU-T (International Telecommunication Union) and the ISO/IEC (International Organization for Standardization/International Electrotechnical Commission). Their efforts are addressed towards two different goals: to transmit video at as small as possible bit rate through standard telephone or mobile networks, leading to a family of H.26x standards (ITU-T), or to support high quality video streaming, obtained from a family of MPEG-x standards (ISO/IEC), where " $x$ " denotes the appropriate suffix. Early video coding standards, such as ITU-T H.261 and ISO/IEC MPEG-1, are designed for a fixed quality level $[1,2]$. Later on, video coding schemes are designed to be scalable, that is, to encode the signal once at highest resolution, but enable adaptive decoding depending on the specific rate and resolution required by a particular application. Such coding schemes permit video transmission over variable bandwidth channels, both in wireline and wireless networks, to store it on media of different capacity, and to display it on a variety of devices ranging from small mobile terminals to high-resolution displays [3-5].

The famous broadcast standard MPEG-2 (which is identical to ITU-T H.262) was the first standard which includes a number of tools providing scalability. The MPEG-4 standard (or, more precisely, a set of various versions of this standard) is multimedia oriented, providing even more flexible scalability tools. Many features, necessary in multimedia, have been introduced: coding in object planes, modelbased coding, including SNR (signal-to-noise ratio) scalability with fine granularity, and so forth. The MPEG-4 standard, Version 10, adopted also from the ITU-T as H.264/AVC (advanced video coding) standard, defocuses two previously defined goals of compression: not demanding the lowest bit rate nor the highest video quality [3]. The idea was to enable rather good quality, almost as good as in MPEG-2, at not obviously the smallest bit rates. Those features make this standard very convenient for video distribution over the Internet. It is expected that forthcoming digital video broadcasting for handheld monitors (DVB-H) will be the first one in 
the broadcasting family accepting the H.264/AVC as a highquality non-MPEG-2 compression.

Video traces of encoded videos have been generated and studied by many authors. Initial study was presented in Mark Garrett's Ph.D. thesis [6]. He has digitized and encoded as M-JPEG (Motion JPEG) the hit movie "Star Wars," and after that analyzed such video material considering the sizes of each encoded video frame, which typically referred to as frame size traces. The studied frame size traces correspond to videos encoded with later MPEG-1 standard without rate control into a single layer. Among different "classical" video traffic metrics, such as mean, coefficient of variation, and autocorrelation, he has used also the rescaled range analysis, or R/S statistic, and the Fourier power spectrum (known as periodogram), for estimating the Hurst parameter, $H$, which describes the long-range dependency (LRD) of the stochastic process. However, note that LRD is only one feature of a "fractal" behavior. For instance, as shown in [7], multifractal analysis allows more precise statistics in describing TCP (Transmission Control Protocol) traffic. Moreover, similar conclusions are derived when analyzing compressed video [8-12]. More precise characterization of modern telecommunication traffic is possible by using multifractal analysis [13].

The Telecommunication Networks Group at the Technical University of Berlin generated the library of frame size traces of long MPEG-4, Version 2, H.261, and H.263 encoded videos [8]. Later on, two groups working at Arizona State University, as well as in acticom $\mathrm{GmbH}$, extended their work to the latest standard H.264/AVC [9$12,14]$. These two groups have been deeply involved with the statistical analysis of video traces. Namely, they calculated different parameters characterizing video traffic and video quality, among them the fractal parameters. Also, they have pointed out the need for multifractal characterization of video traces, but left these investigations for future work [9].

Analyses of encoded video traces have been preformed also in [15-17], with special attention to fractal and multifractal characterization of M-JPEG and MPEG-1 encoded movie "Star Wars." Later on, we have studied multifractal features of video compressed material available at [8], and performed different analysis over them [18, 19].

This paper considers the fractal and multifractal nature of video traces encoded according to the ITU-T H.264/AVC standard. The paper is organized as follows. Section 2 gives the brief review of fractal and multifractal analyses with special attention to their application in characterization of compressed video. Simulation results are presented in Section 3. We have analyzed long video traces of "Starship Troopers" movie compressed according to H.264/AVC standard and publicly available at [14]. The results are compared to those obtained when the same sequences are compressed by other coding standards, such as H.261, H.263, and MPEG-4, Version 2, of different quality. Some conclusion remarks and suggestions for future work are given in Section 4.

\section{FRACTAL AND MULTIFRACTAL NATURE OF VIDEO TRACES}

\subsection{Long-range dependency of video traces}

For one-dimensional signals the description of the longrange dependency in data (i.e., the fractal nature of the process) may be derived from the Hurst index, $H$ [20]. It was shown that pure random process (e.g., Brownian motion) is characterized by $H=0.5$. In this case there is no correlation between incremental signal changes [20]. If $0.5<H<1$, there is a positive correlation between incremental changes, that means, if the process increases in some time interval, then it tends to continue to increase in the nearest interval, and vice versa if it decreases - being thus self-similar, that is, exhibiting the LRD behavior. This tendency is as strong as the Hurst index is closer to unity. Conversely, if $0<H<0.5$, the opposite is true. Then the negative correlation between the increments (or a short-range dependency (SRD)) arises and the system has a tendency to oscillate. The Hurst index can be estimated in several ways: through R/S statistics, from periodogram, and/or IDC (index of dispersion constant) method, by using wavelet estimator [21], or indirectly, through the fractal dimension.

Figure 1 illustrates the LRD behavior of the process. This figure gives the frame sizes, in bytes per frame, as a function of the frame number. (a) corresponds to one hour of the movie "Starship Troopers" with 25 frames per second (90,000 frames) compressed according to the ITU-T H.264/AVC standard, with quantization scale $q_{p}=15$ [14]. By zooming a part of a whole trace, for instance, from 50,000 to 53,000 frames (Figure 1(b)) and further, from 51,370 to 51,550 frames (c) the LRD behavior of compressed video is visually approved, because the shape of all sequences remains similar, irrespective of the time scale.

Numerical evaluation of the LRD behavior of the signal as in Figure 1 is performed through Hurst indices. The $\mathrm{R} / \mathrm{S}$ statistic is computed for logarithmically spaced aggregation level $k$, by considering different starting points. Plotting $\log (R / S)$, as a function of $\log (k)$ gives R/S diagram (also referred to as pox diagram of $R / S$ ) [9]. The Hurst index is estimated as a slope of linear regression line. This procedure is illustrated in Figure 2(a) where R/S plot for first 10,000 frames of "Starship Troopers" movie, as in Figure 1, assuming aggregation level 100 and setting 7 different starting points (labeled by different marks), is depicted. From the slope of linear regression line we estimated $H=0.89768$.

Another way we used for estimating the Hurst index was the periodogram method. When plotting periodogram in a log-log plot, the Hurst index may be estimated from a slope of least square regression as $H=(1-$ slope $) / 2$. The periodogram of the same sequence of 10,000 frames is depicted in Figure 2(b), from which the Hurst index is estimated as $H=0.82934$. Note that values of $H$ indices obtained from different estimators may be different, as obtained in considered case. This is in accordance with the results already reported in literature, for instance in $[6,20,21]$. Note that, for process with high periodicity, the estimated $H$-index may be even greater than 1, despite its LRD feature [9]. Removing 


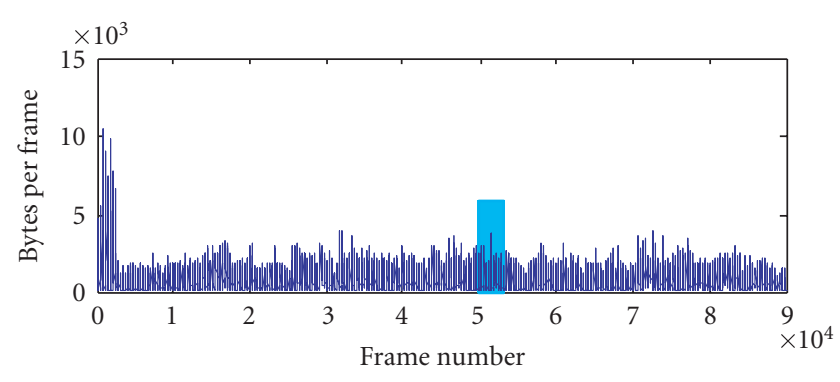

(a)

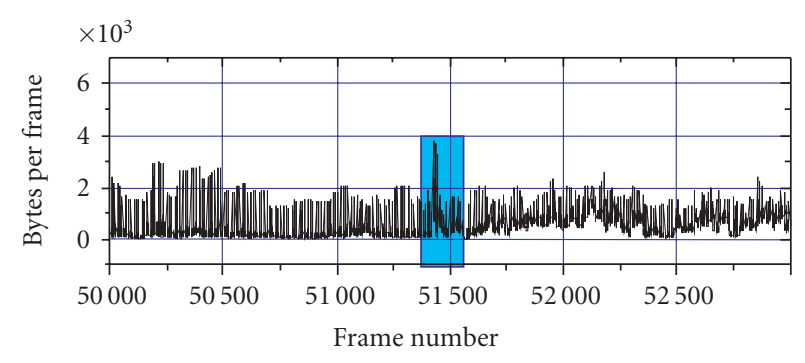

(b)

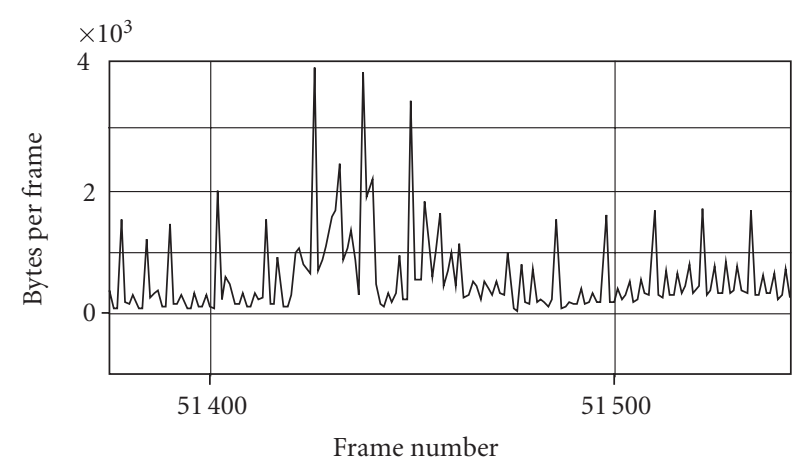

(c)

Figure 1: A part of "Starship Troopers" video traces compressed according to H.264/AVC standard (quantization scale $q_{p}=15$ ), and its zoomed parts ((b) and (c)).

the periodicity from the signal and then applying the Hurst estimator, more useful information may be obtained [22].

In Table 1 the Hurst indices for "Starship Troopers" video traces of different lengths (described by number of video frames) compressed according to H.264/AVC standard are listed. Hurst indices are estimated from periodograms, for three different quantization scales, $q_{p}$. In all cases the LRD property is approved $(0.5<H<1)$, that is, considered video traces are self-similar. Also, the Hurst index varies with the quantization scale $q_{p}$, that is, with the compression rate. Digitized "Starship Troopers" movie, as well as other digital videos, exhibits inherent fractal property (or isolated fractal behavior). Such property was obtained from the process itself, without any interaction with network or some other source of variability [21]. Certainly, when sending such a video over real network, traffic conditions influence the signal and may change its Hurst index, both increasing or decreasing it, depending on particular case. For instance, when

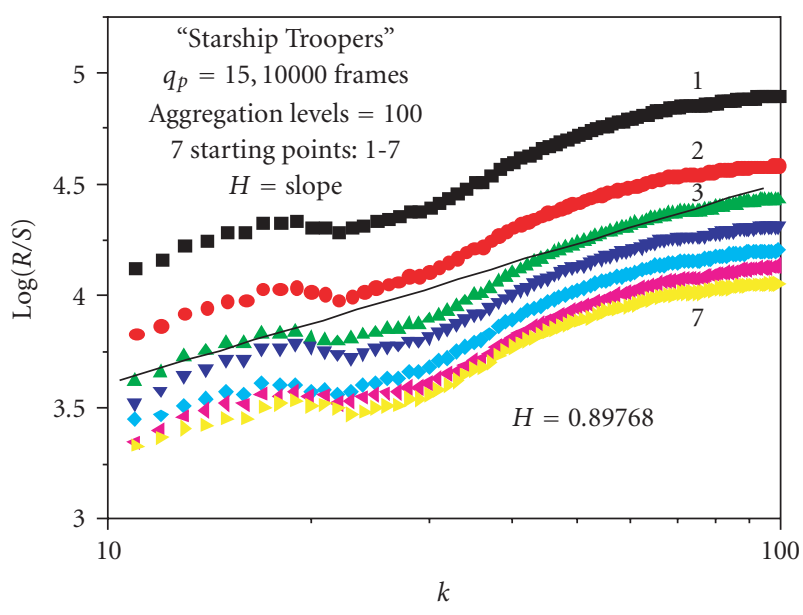

(a)

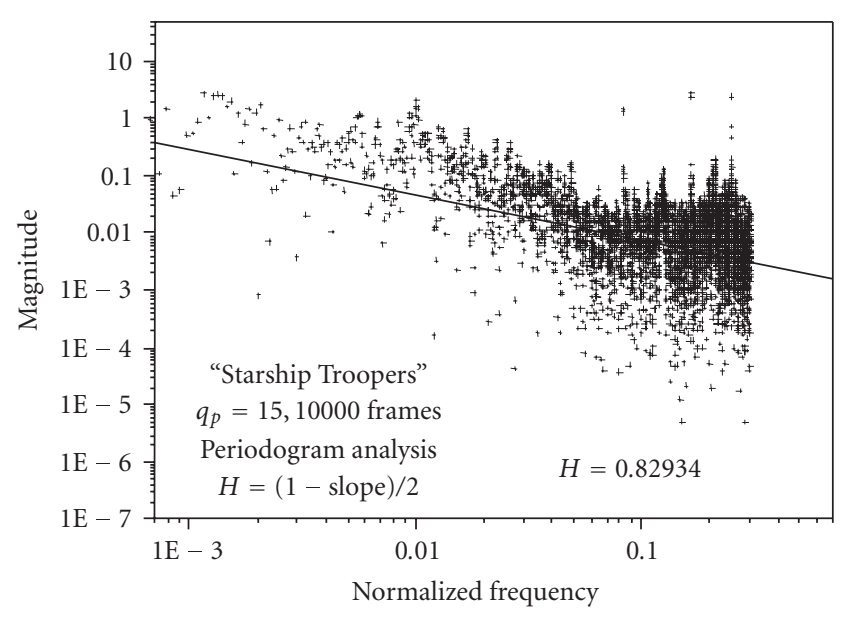

(b)

FIgURE 2: (a) R/S plot and (b) the periodogram for first 10000 frames of the "Starship Troopers" movie, compressed according to H.264AVC with quantization scale $q_{p}=15$ [14].

using neural network scheduling in packet switching node [15], outgoing traffic tends to be less fractal than the incoming one-the Hurst index decreases approaching to 0.5 (random walk process) $[23,24]$. In this paper the influence of external sources of variability is not considered.

\subsection{Multifractal analysis of video traces}

The Hurst index is one of the possible descriptors of fractal behavior. Fractal structures may be evaluated through their fractal dimension as well. Practical and very often used technique for estimating fractal dimension is box counting [25-27] In this method we cover observed structure with $d$ dimensional boxes with size $\varepsilon$, and count the number of occupied boxes, $N(\varepsilon)$. Fractal dimension is then estimated as

$$
D_{f}=-\lim _{\varepsilon \rightarrow 0} \frac{\ln (N(\varepsilon))}{\ln (\varepsilon)} \text {. }
$$


TABLe 1: Hurst indices for "Starship Troopers" video traces of different lengths (described by number of video frames) for different quantization scales, $q_{p}$, or compression ratio, CR.

\begin{tabular}{l|lcc}
\hline $\begin{array}{l}\text { Video trace } \\
\text { length: }\end{array}$ & \multicolumn{3}{|c}{ Hurst indices for different } \\
number of & quantization scales, $q_{p}$, or compression ratio, CR \\
\cline { 2 - 4 } frames & $q_{p}=5$ & $q_{p}=15$ & $q_{p}=25$ \\
\hline 500 & 0.7802 & $\mathrm{CR}=15.9$ & $\mathrm{CR}=79.0$ \\
800 & 0.78637 & 0.94298 & 0.75985 \\
1000 & 0.79554 & 0.78477 & 0.80582 \\
3000 & 0.80417 & 0.79481 & 0.80701 \\
5000 & 0.80282 & 0.8041 & 0.79143 \\
10000 & 0.81522 & 0.80181 & 0.69483 \\
20000 & 0.81245 & 0.82934 & 0.80202 \\
30000 & 0.81538 & 0.83263 & 0.8018 \\
50000 & 0.81421 & 0.84327 & 0.80451 \\
70000 & 0.81219 & 0.81227 & 0.81262 \\
90000 & 0.8953 & 0.84631 & 0.81308 \\
\hline
\end{tabular}

It was shown that for one-dimensional signals fractal dimension and Hurst index relate as [26, 27]

$$
D_{f}=2-H \text {. }
$$

Fractals may be generated artificially by applying some exact rule. Such structures are known as deterministic (or, mathematical) fractals. Since they are composed of parts whose smaller scales replicate exactly their larger ones, up to infinity, they have the same fractal dimension in all scales, and consequently are referred to as exact self-similar, or monofractals. A lot of such structures are known, for instance, Cantor sets, Koch's curves, Sierpinski gasket and carpet, and so forth, [25-27].

Instead, a variety of natural objects, structures, and phenomena are characterized by self-similarity in some statistical way: the reproduced detail is not an exact copy of the previous. Such objects are referred to as random fractals. Also, natural fractals are not self-similar over all scales. There are both upper and lower size limits, beyond which a structure is no longer fractal. Upon closer examination of random fractals it is possible to recognize subsets with their own fractal dimension which varies with the observed scale; so, they may be referred to as multifractals (MF). We can assume such structures as fractals embedded within fractals. For describing them more sophisticated mathematical quantities are necessary $[28,29]$. Just as classical geometry is unable to accurately depict many natural structures, traditional fractal analysis techniques may also fall short in fully describing natural patterns.

The quantitative description of multifractal property can be derived in several ways [7, 28-31]. Very often, the procedure starts with finding the noninteger exponent $\alpha$, known as the Hölder exponent, describing the pointwise singularity of the object, and then deriving the distribution of this quantity, known as the multifractal spectrum, $f(\alpha)$, as will be briefly reviewed.

Let the structure $S$ be divided into nonoverlapping boxes $S_{i}$ of size $\varepsilon$ such that $S=\bigcup_{i} S_{i}$. Each box $S_{i}$ is characterized by some amount of measure, $\mu\left(S_{i}\right)$. An appropriate parameter suggested to the MF analysis is defined by

$$
\alpha_{i}=\frac{\ln \left(\mu\left(S_{i}\right)\right)}{\ln (\varepsilon)}
$$

which is denoted as the coarse Hölder exponent of the subset $S_{i}$. If $\varepsilon$ tends to zero the coarse Hölder exponent approaches to limiting value $\alpha$ at observed point

$$
\alpha=\lim _{\varepsilon \rightarrow 0}\left(\alpha_{i}\right) .
$$

Parameter $\alpha$ depends on the actual position on the fractal and describes local regularity of the structure. In the whole structure there are usually many boxes with the same parameter $\alpha_{i}$. We may find the distribution of this quantity over the subsets characterized by $\alpha_{i}$, as

$$
f_{\varepsilon}\left(\alpha_{i}\right)=-\frac{\ln \left(N_{\varepsilon}\left(\alpha_{i}\right)\right)}{\ln (\varepsilon)},
$$

where $N_{\varepsilon}\left(\alpha_{i}\right)$ is the number of boxes $S_{j}$ containing particular value of $\alpha_{i}$. From (5) one can obtain the limiting value

$$
f(\alpha)=\lim _{\varepsilon \rightarrow 0}\left(f_{\varepsilon}(\alpha)\right),
$$

known as the Hausdorff dimension of the distribution of $\alpha$, or the MF spectrum. This function describes the global regularity of observed structure [7, 28-33]. Note again that box counting is only one among several different methods for estimating the MF spectrum, but due to its simplicity and fast computing procedure this method is very often used [28-31]. Irrespective of particular technique for deriving MF quantities $\alpha$ and $f(\alpha)$, they describe both local and global regularities of the process under investigation. Consequently, MF analysis may be used in a broad class of signal processing problems, as a robust method for describing and/or extracting some features probably hidden in large amount of data.

For instance, it was shown that for TCP traffic the LRD indices are not quite appropriate for describing such process. By analyzing TCP traffic at Berkeley, Riedi and Vehel [7] shown that significant differences between incoming and outgoing traffic flows may be derived from the shapes of their multifractal spectra although both traffics are characterized by almost the same Hurst indices.

From the R/S diagram in Figure 2(a) qualitative description of the multifractal nature of this process may be inferred. As noted earlier, the Hurst index is estimated as the slope of linear regression line of R/S diagram. From Figure 2(a) it is evident that the slope differs at different aggregation levels, indicating the local variation of $H$ indices, thus "Starship Troopers" movie compressed according to the ITU-T H.264/AVC is multifractal. Similar conclusion was derived also when analyzing video sequences compressed according to H.261, H.263, and MPEG-4 standards [16-19]. 
Very intensive growth of multimedia applications, where compressed video has a dominant role, has been changing the nature of teletraffic, in general. From POTS (plain old telephone services) networks, where the traffic was successfully described by Poisson distribution, the new teletraffic changes the statistics, typically exhibiting high bit rate variability (burstiness) as well as LRD (or self-similarity) $[20,22]$. Multifractal analysis, being capable to perform both local and global features of the process under investigation, seems to be more appropriate for analysis of compressed video and thus for analyzing modern teletraffic. Results obtained from MF analysis of compressed video may contribute to more accurate modeling of modern teletraffic and multimedia. Moreover, by appropriate choice of method for finding multifractal quantities $\alpha$ and $f(\alpha)$ it may be possible to establish one-by-one correspondence between points in signal space and in MF space permitting thus the "inverse" multifractal analysis: finding parts in signal space having particular value of $\alpha$ and/or $f(\alpha)$ [30-33]. For instance, from once derived pair $(\alpha, f(\alpha))$ of video trace, we may extract frames with high (or low) local fractal behavior (characterized by high (or low) $\alpha$ values, resp.) and/or extract frames, having particular value of $f(\alpha)$, which are globally rare events (having low $f(\alpha)$ ) or are frequent in video trace (high $f(\alpha)$ ). In this way we can describe more completely the nature and structure of observed video traces. Similar procedure was already applied in image processing, for instance, in [30-33].

\section{SIMULATION RESULTS}

We have analyzed long "Starship Troopers" movie video traces (one hour of movie with 25 frames/second, containing 90,000 frames), compressed according to H.264/AVC standard and publicly available at [14]. Frame size traces are analyzed from the fractal and multifractal points of view. The results were compared to those derived for the same sequences compressed according to other coding standards, H.261, H.263, and MPEG-4 Version 2, available at [8]. For reasons of interoperability and low cost, video material was assumed in QCIF (quarter common intermediate format) resolution format $(144 \times 176$ pixels per frame). Fractal behavior in video sequences was investigated through the Hurst index, determined from R/S diagram and periodogram, as described in Section 2.1. Multifractal quantities $\alpha$ and $f(\alpha)$ were estimated by applying histogram method, already developed in [32]. The choice of a method is motivated by the fact that it retains high-frequency components in MF spectrum permitting sharp distinction between fine details emphasizing thus the singularities. In addition, this method enables inverse multifractal analysis, as described in Section 2. Note that publicly available algorithms, for instance, the method of moments suggested by Chhabra and Jensen [34] and embedded in software MATPACK [35], as well as the method using Legendre measure, used in software FracLab [36], produce good-looking but very smooth MF spectra, where some specific information may be hidden.

Figure 3(a) represents the MF spectra derived for the "Starship Troopers" movie for all available cases from database [14], that is, for all quantization scale parameters: from $q_{p}=1$ to $q_{p}=31$. Although it is difficult to distinguish particular spectra in Figure 3, because plots are erratic and interwoven, several fundamental conclusions may be derived. First, for low $q_{p}\left(q_{p}=1,5,10\right)$ the MF spectrum is narrow (exhibiting mainly LRD behavior), concave and almost symmetrical around its maximum near $\alpha=1$. Higher values of $q_{p}$ produce broader spectra indicating to higher multifractal nature. Note that quantization scale parameter relates to a compression ratio, $\mathrm{CR}$, expressed as the ratio between the number of bytes of uncompressed versus compressed video. As a reference, the values of CR for video traces analyzed in this paper are listed in Table 2.

Furthermore, as $q_{p}$ increases the spectra become more asymmetrical (in this case right-sided, i.e., going to higher $\alpha$ ), having more local maxima and local singularities.

Previous investigations of different processes [7] have shown that pure concave (parabola-like) MF spectrum is obtained for multiplicative process. Failure of being concave is a sign that observed process is not pure multiplicative one. For instance, if the signal is composed by additive components, extra parabola-shaped curves would appear in the spectrum. Diagrams presented in Figure 3 exhibit such behavior, when increasing the quantization scale, or compression ratio.

The MF spectrum of "Starship Troopers" movie is almost concave, Figure 3(a), for quantization scale $q_{p}=1$, indicating to the multiplicative nature of the process. However, additional small parabolas arise at both sides of the spectrum. This is the sign of the existence of additive components, but these events are rare (having small $f(\alpha)$ values) in a whole movie. Remind that the sequences in H.264/AVC video, as well as in MPEG-4, consist of I, P, B (intra-coded, predictive, bidirectional) frames within the GOP (group of picture) structured as IBBPBBPBBPBBI, in coding order. In order to find the sources of irregularities in MF spectra, we investigated traces extracted from a whole movie, containing only I, P, or $\mathrm{B}$ individual frames, for all quantization scale parameters as for a whole video. Corresponding MF spectra are depicted in Figures 3(b)-3(d).

For I-frames MF spectra, Figure 3(b), retain almost concave shape at all quantization scales, with very small singularities. Bearing in mind that those frames are intra-coded, exploiting only spatial redundancy between pixels within the same frame, such a feature is expectable, because I-frames have the smallest compression rate and smallest variability in size versus quantization scale.

On the contrary, inter-coded frames, $\mathrm{P}$ and $\mathrm{B}$, exploit mainly the temporal redundancy. In addition, these frames contain usually small amount of new information at the positions from which objects start to move. The relevant content of these frames will be changed depending on the quantization scale. In this way the additional compression is obtained forcing the smaller frame sizes, producing more variability (the motion vectors information is kept unchanged). For small quantization scales $q_{p}$ (up to 10), that is, small compression rates (up to 7), MF spectra of $\mathrm{P}$ and $\mathrm{B}$ video traces are of rather regular concave shape, slightly broader than corresponding MF spectra of I frames. But as compression rate 


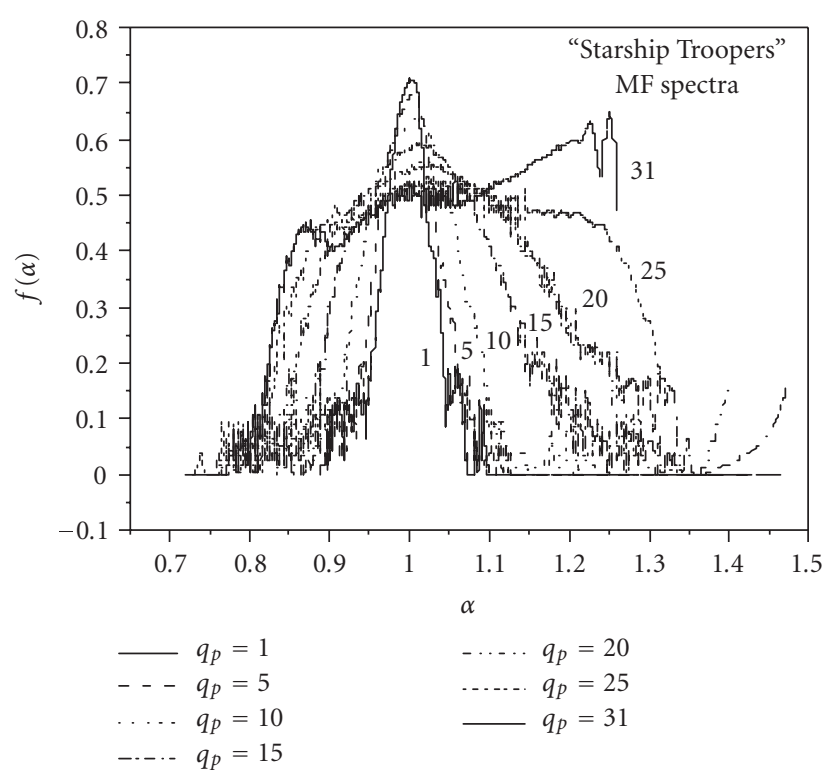

(a)

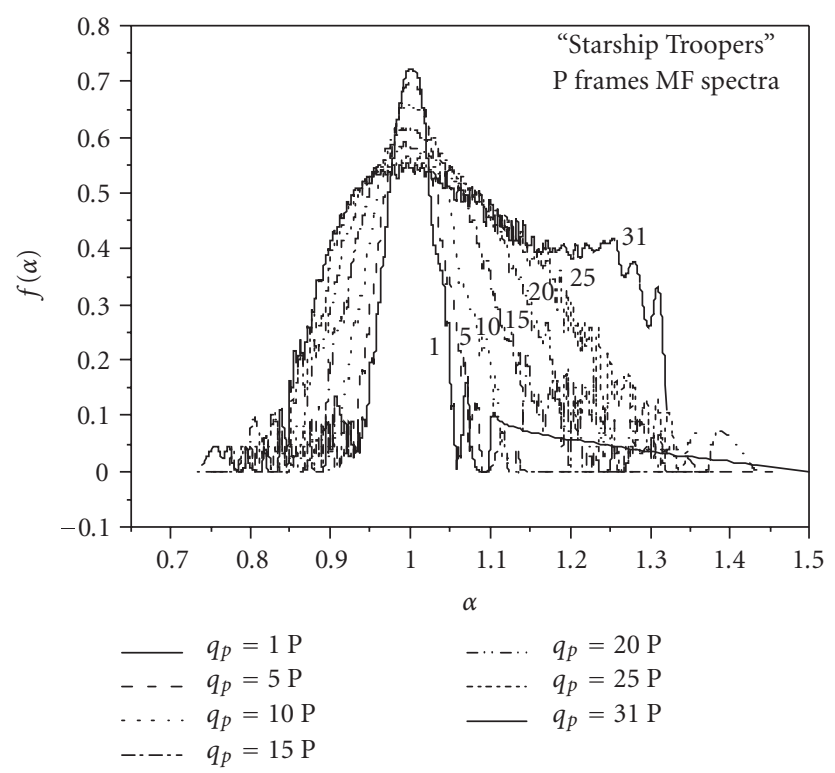

(c)

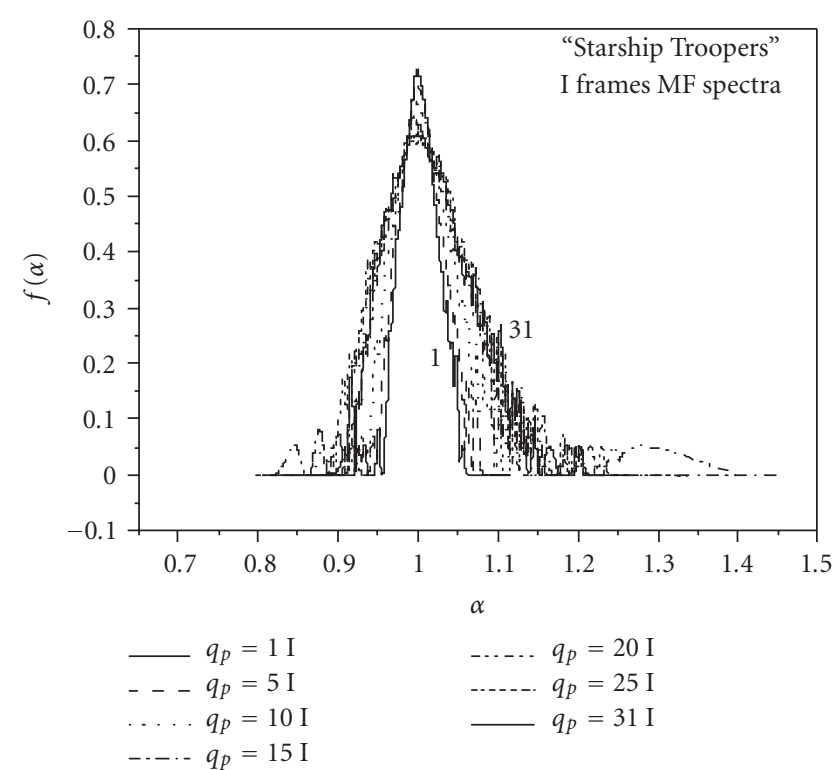

(b)

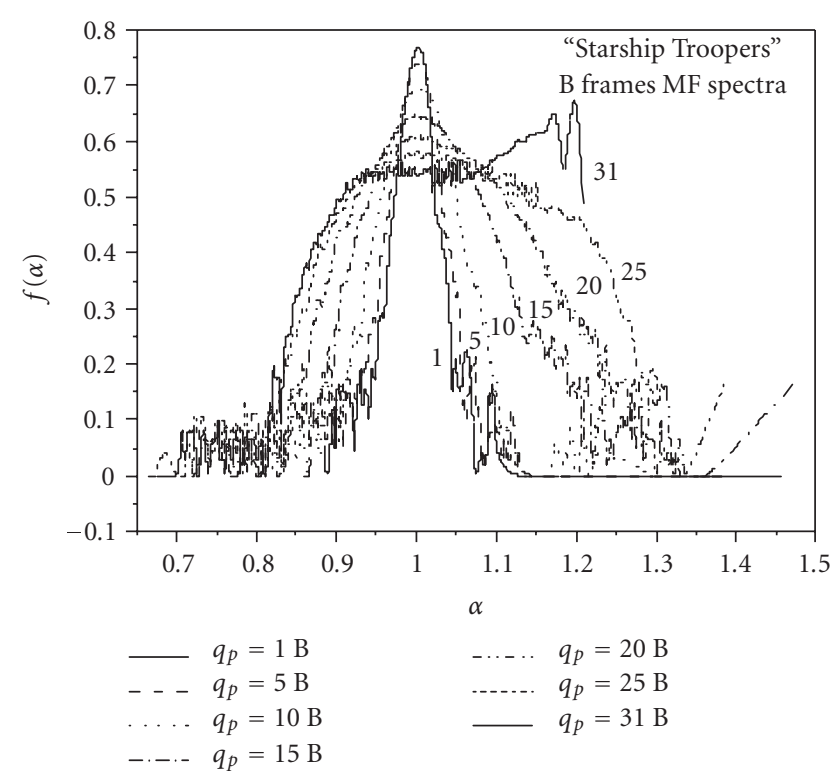

(d)

Figure 3: Multifractal spectra for H.26L “Starship Troopers” video traces: (a) all frames; (b) I frames only; (c) P frames only; (d) B frames only.

increases, $\mathrm{P}$ and $\mathrm{B}$ spectra become more broader and more irregular indicating the higher multifractal nature of these traces. Comparing Figure 3(a) to 3(d), one can conclude that the whole video is composed of additive components I, P, and $B$, and that $B$ frames have the greatest influence on the whole MF spectrum, particularly at higher quantization scales.

For better comparison of I, P, and B traces and their influence on the whole movie, we choose three quantization scales $q_{p}, q_{p}=5,15$, and 25. Their MF spectra are depicted in Figures 4(a)-4(c) from which three main conclusions may be better clarified. By increasing the quantization scale all the three spectra are extended, become unsymmetrical (rightsided), and point out more additive components. The MF spectra of I frames are less changeable with $q_{p}$, while the opposite is with B frames. Migration to the right side of MF spectra at higher $q_{p}$ indicates the increasing of the local fractal behavior of the process.

We also compared the traces of H.264/AVC and MPEG4, Version 2 (single layer, too), of the same movie. The GOP structures of those sequences were the same, with $q_{p}=10$ in both cases. By applying the procedure as above we calculated the MF spectra for a whole MPEG-4 trace 
TABle 2: Compression ratio, CR, expresed as the ratio between the number of bytes of uncompressed and compressed videos.

\begin{tabular}{l|cc}
\hline Coding standard & $q_{p}$ & CR \\
\hline & 1 & 2.21 \\
& 5 & 3.50 \\
& 10 & 7.00 \\
H.264/AVC & 15 & 15.91 \\
& 20 & 35.75 \\
& 25 & 78.96 \\
& 31 & 189.80 \\
\hline MPEG-4 V2 & 10 & 37.68 \\
H.261 VBR & $\mathrm{x}$ & 17.47 \\
H.263 VBR & $\mathrm{x}$ & 21.50 \\
\hline
\end{tabular}

and for separated I, P, and B traces. Corresponding spectra are depicted in Figure 5. The MF spectrum of MPEG-4, Figure 5(a), is wider and more regular (with less variability) than that of H.264/AVC, and the same conclusion is valid for MF spectra of separated I, P, and B frames. Also, one can observe that $\mathrm{B}$ traces are wider and have greatest influence on the whole MF spectrum in both cases (MPEG-4 and H.264).

Since $q_{p}$ relates to compression ratio, we also compared the H.264/AVC, $q_{p}=10$, with MPEG- $4, q_{p}=20$, since those sequences have (almost) the same compression ratio (35.75 and 37.67, resp.). Corresponding spectra, depicted in Figure 6, are almost identical except at both ends, particularly at high values of $\alpha$, where that of H.264/AVC exhibits more variability.

It is known that the maximum of MF spectrum corresponds to the fractal dimension of the whole structure [7] describing most frequently events in the structure. By examining MF spectra from Figure 3(a), close to their maxima, the plots as in Figure 7(a) are obtained. As we can see, by increasing the quantization scale maxima migrate rightward (to higher $\alpha$ ) while corresponding values of $f(\alpha)_{\max }$ become lower. Such behavior indicates that higher $q_{p}$ (slightly) increases local fractal behavior of most frequently events but the number of these events decreases. From the whole MF spectra we already concluded that higher compression rate leads to broader MF spectra and more singularities. The statistics of the compressed video are changed.

For comparison purposes we analyzed the same video traces as discussed previously, by other methods and available computing tools, such as the method of moments [34], embedded into the MATPACK software [35], as well as the method using Legendre spectrum embedded into the FracLab software [36]. Corresponding MF spectra are depicted in Figures 8 and 9. Lower diagrams show zoomed details around maxima.

Global shapes of these diagrams are similar to ours: as $q_{p}$ increases spectra become wider and right-sided, with rightward shifting of maxima. Both diagrams exhibit high smoothness, but fine details are missed. Also, both diagrams have the parts with negative $f(\alpha)$, which correspond to regions where the probability of observing $\alpha$ decreases too fast with the grid size $\varepsilon$ [7]. In our approach in these regions
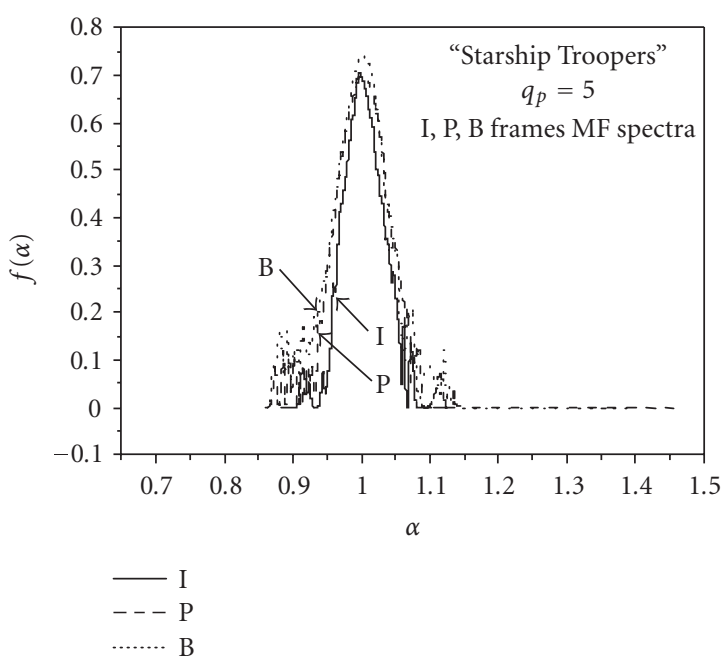

(a)
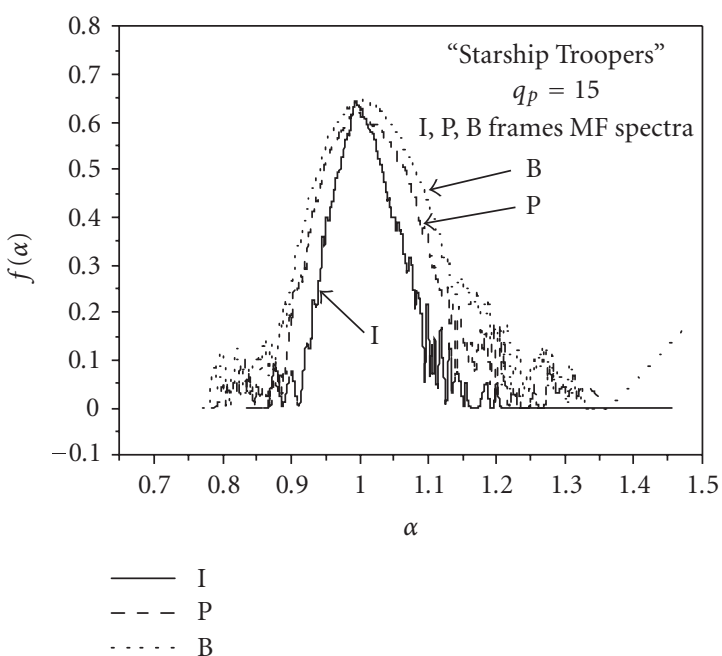

(b)

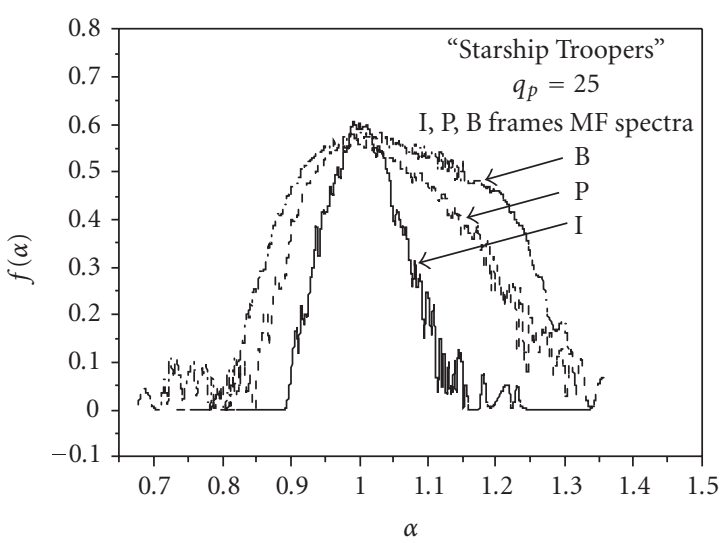

$$
\begin{aligned}
& -\mathrm{I} \\
& ----\mathrm{P} \\
& \ldots-.
\end{aligned}
$$

(c)

FIgUre 4: Multifractal spectra for I, P, B frames and for different quantization scales: (a) $q_{p}=5$; (b) $q_{p}=15$; (c) $q_{p}=25$. 


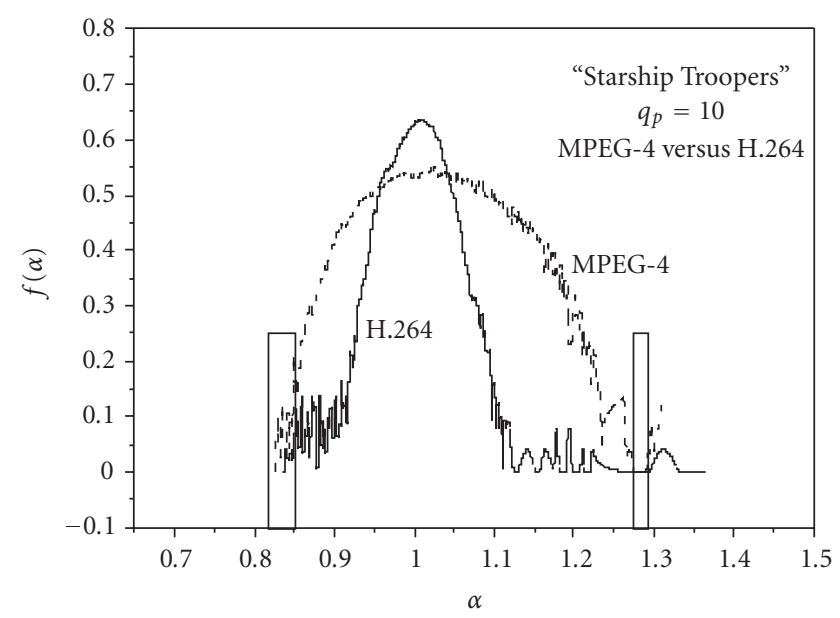

(a)

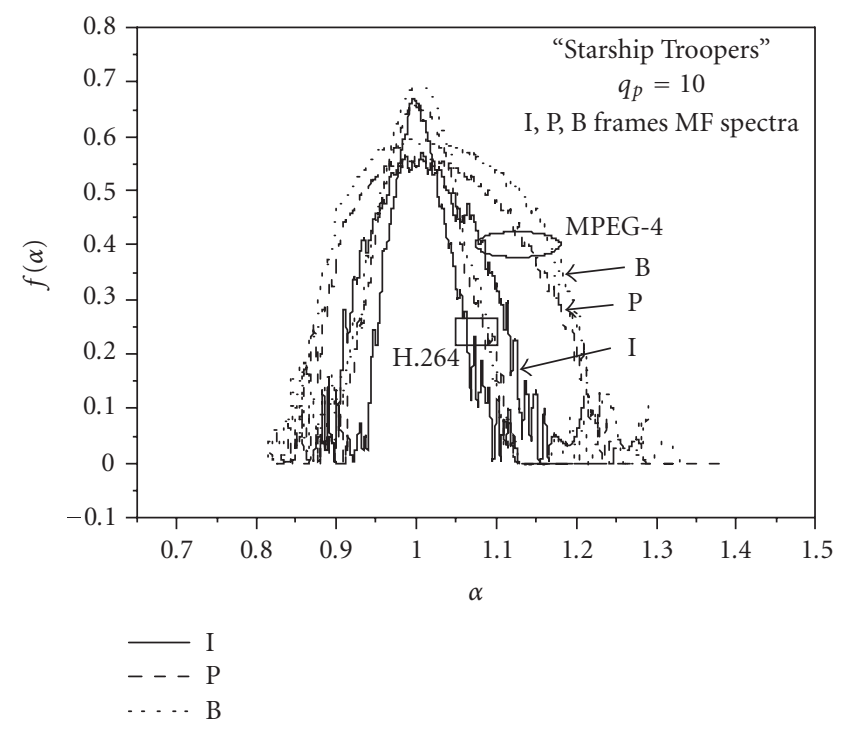

(b)

Figure 5: (a) H.264/AVC versus MPEG-4 multifractal spectra; (b) separated I, P, B.

we estimated high variability of MF spectrum, indicating to additive components. In Figure 9 all diagrams have maxima with the same value of $f(\alpha)_{\max }=1$, but this is a consequence of normalization.

The compression rate has strong influence on fractal and multifractal nature of compressed video, which we have approved by analyzing the same movie compressed by the H.261 and H.263 standards, without output rate control (known as variable bit rate), leading to low bit rates. Although these two compression techniques are not easily comparable to the H.264/AVC (the frame structures are different because both standards have no GOP and H.263 using I, P, and $\mathrm{PB}$ frames, instead of $\mathrm{B}$ frames), from the shape of MF spectra, Figure 10, it is evident that higher compression rate (H.263var) leads to broader MF spectrum.

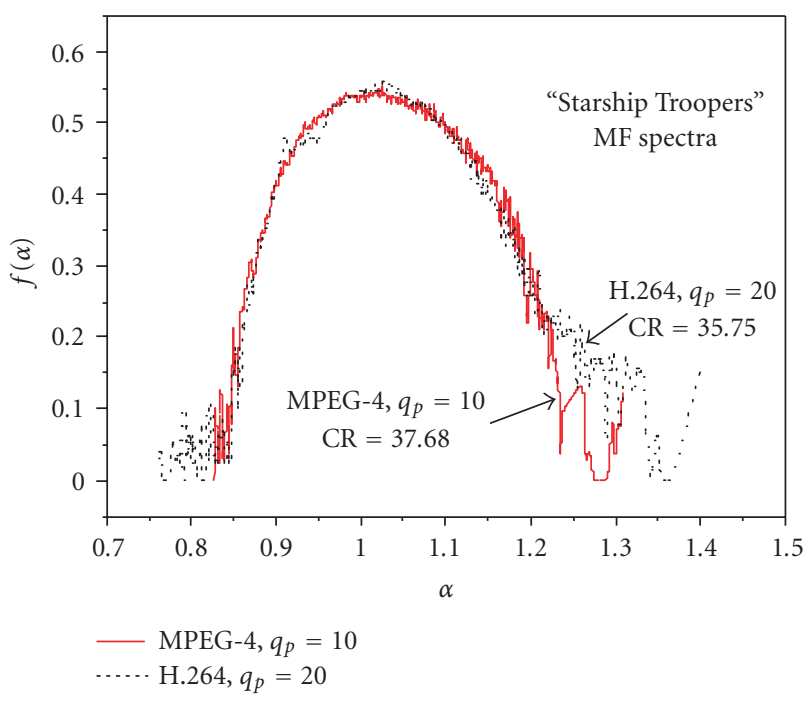

FIgUre 6: Multifractal spectra of H.264/AVC, $q_{p}=10$ and MPEG-4, $q_{p}=20$.

We already noted that by applying inverse multifractal spectra one can extract some specific information from a whole signal. Such a possibility will be approved through several examples. Let us observe, first, the "Starship Troopers" H.264/AVC with $q_{p}=15$, as in Figure 1 and redrawn in Figure 11(a). Its MF spectrum is depicted in Figure 3(a) and separated spectra for I, P, and B frames in Figure 4(b). From these spectra we recognize additive components (singularities) at low $\alpha$. By choosing frames having $\alpha$ in the range from 0.7701 to 0.7703 , irrespective of the range for $f(\alpha)$, extraction of only few frames is obtained, as depicted in Figure 11(b). By zooming the range from 50,000 to 53,000 frames the closer position of those frames is possible, as already depicted in Figures 1(b) and 1(c). As it can be seen, those are frames with sharp change of content, probably because of the change in the movie scene (corresponding to new shots in video sequence).

As a second example we will observe the same movie but compressed with MPEG-4 Version 2 coding standard with $q_{p}=10$. The whole one-hour trace is depicted in Figure 12(a). From Figure 5, where its MF spectrum is presented, an interesting "hole" arises at high $\alpha$, around 1.28. By choosing $1.271<\alpha<1.285$, as indicated into the right box in Figure 5(a), and applying inverse MF analysis we extracted several very short frames: of the length of about 70 bytes, as depicted in Figure 12(b). In this range of $\alpha$ the value of $f(\alpha)$ is almost zero, indicating the extremely rare events, but locally, these frames highly differ from surrounding, which will be more visible when zooming the part of video trace around 40,300 and 66,000 frames.

On the contrary, when choosing singularities from the left side of MF spectrum, values of $\alpha$ between 0.82 to 0.85- see left box in Figure 5(a), we extracted frames as in Figure 12(c). These frames have similar meaning as those in Figure 11(b): they are the largest singular frames in the whole video. 


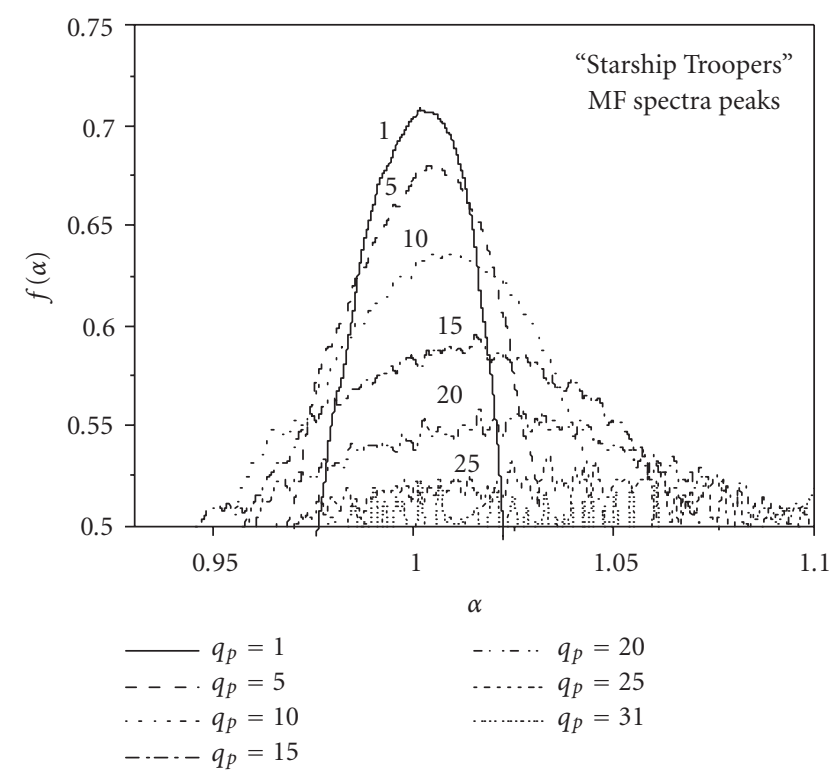

(a)

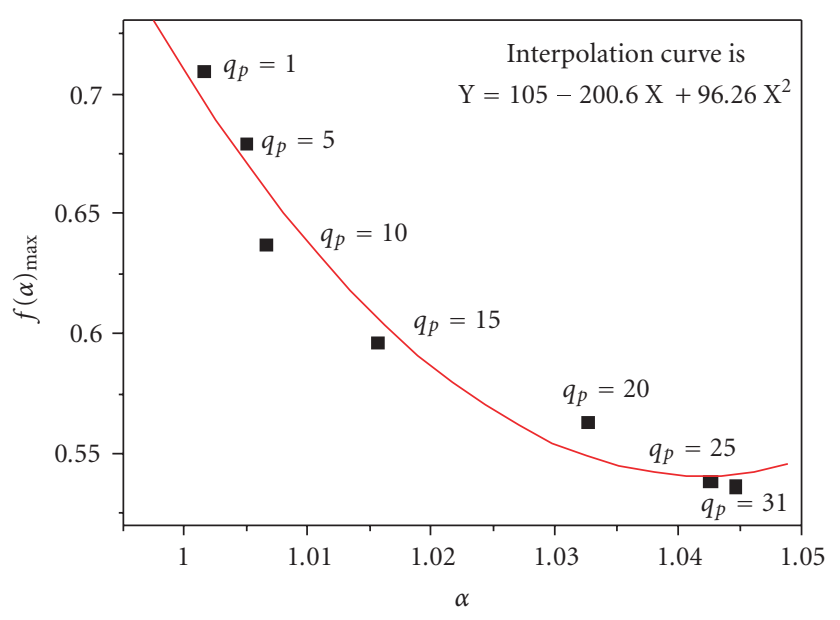

FIGURE 7: (a) Part of multifractal spectra for H.264/AVC video traces around their maxima. (b) Maxima of MF spectra for H.264/AVC video traces and interpolation curve.

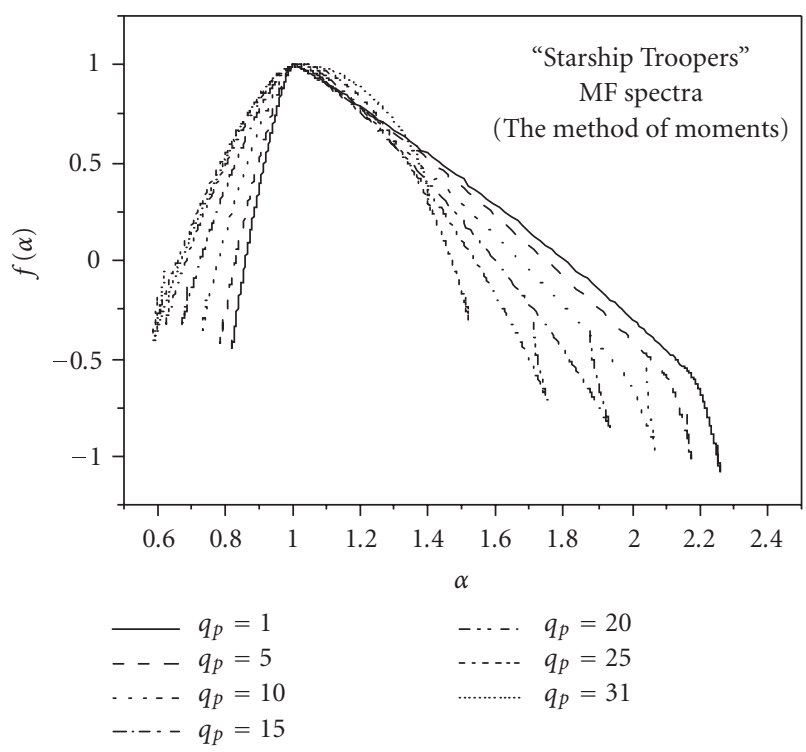

(a)

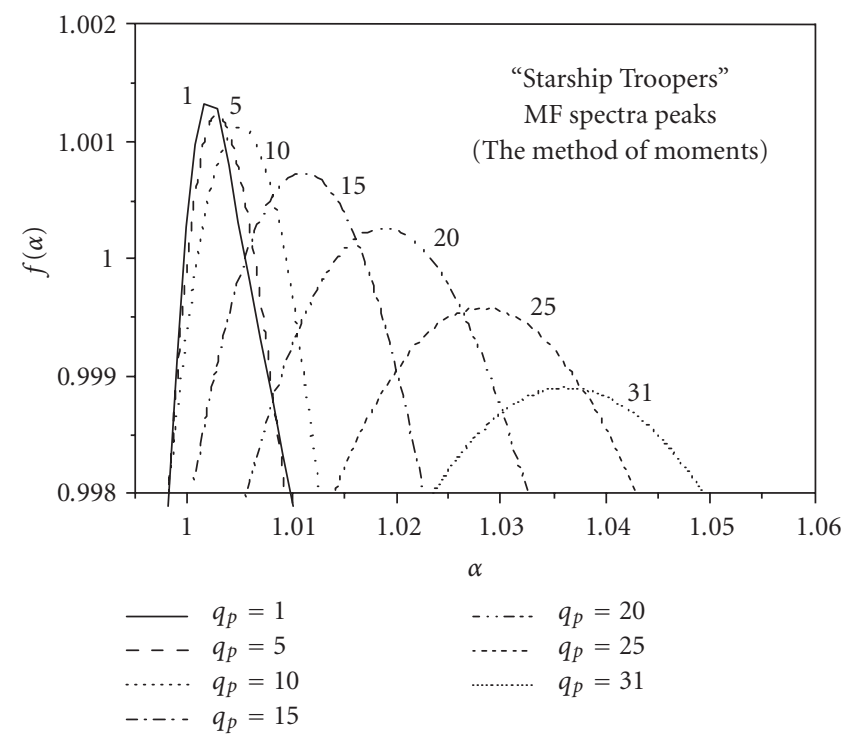

(b)

FIgURE 8: (a) The MF spectra of video traces "Starship Troopers" obtained when applying method of moments embedded into the package MATPACK [35], and (b) their zoomed details around maxima.

\section{CONCLUSIONS}

Long video traces of "Starship Troopers" movie compressed according to H.264/AVC standard have been analyzed. The motivation of this work lies in the expectation that this coding standard will be used in digital video broadcasting for handheld monitors providing high-quality video with low bit rates. Also, since this standard enables rather good quality of transferred video, almost as good as in MPEG-2 but with significantly smaller bit rates, it is very convenient for video distribution over the Internet. Among different statistical parameters (frame sizes versus time, aggregated frame sizes, frame size histogram, i.e., the distribution of frame sizes, mean, coefficient of variance, peak/mean value, etc.), 


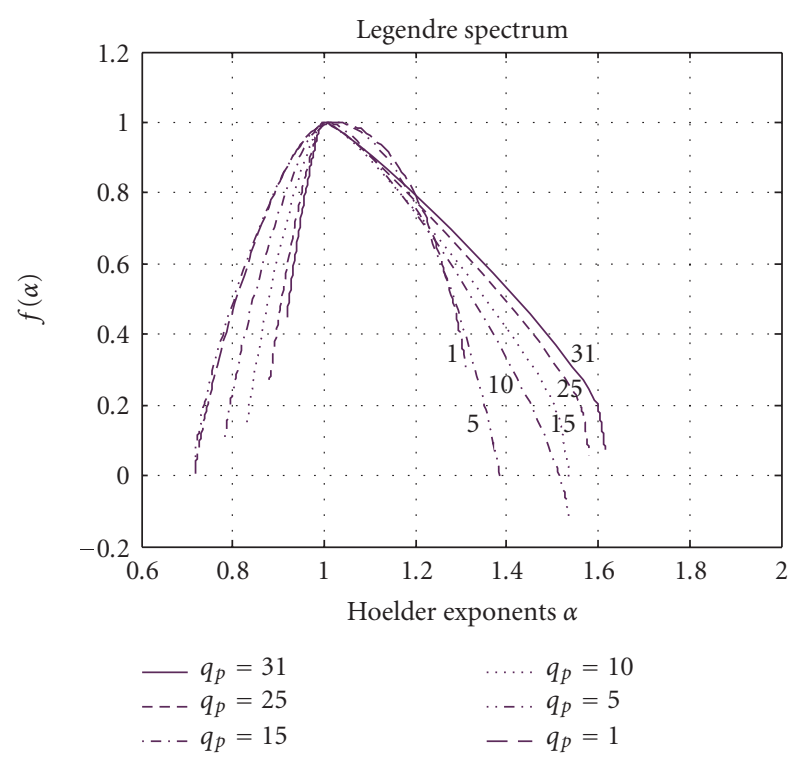

(a)

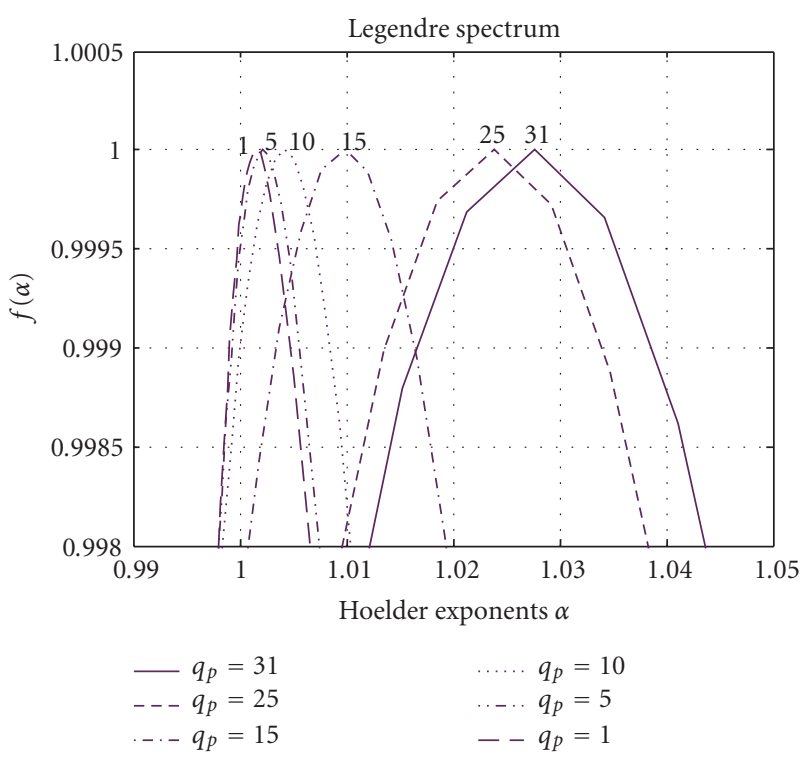

(b)

Figure 9: The MF spectra of video traces "Starship Troopers" obtained when applying Legendre method embedded into the package FracLab [36], and their zoomed details around maxima.

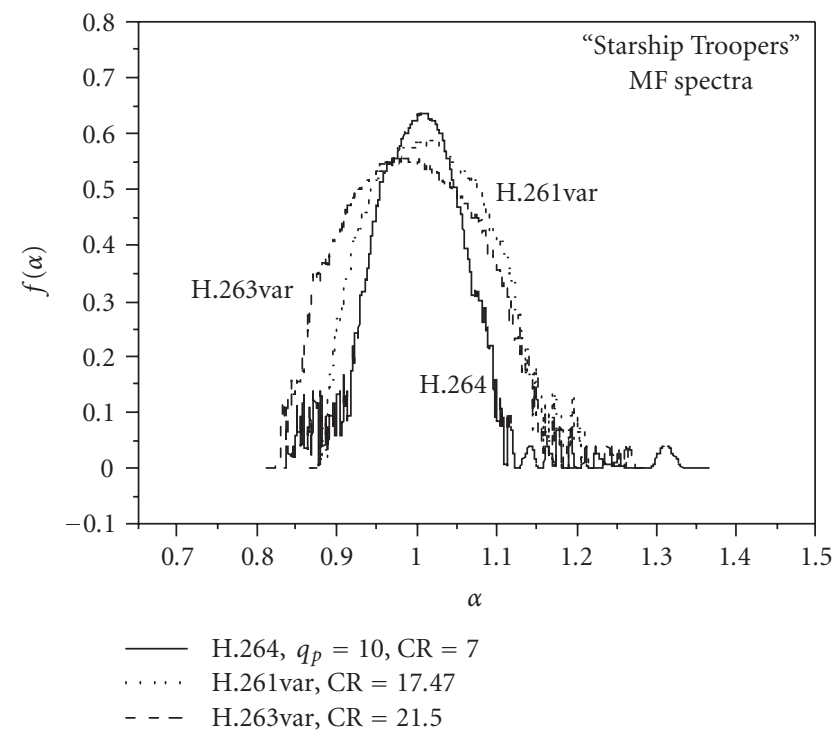

FIGURE 10: MF spectra for different compression standards.

which have been examined by other researchers, we concentrated our attention mainly on fractal and multifractal analyses. Modern teletraffic exhibits long-range dependency, which may be evaluated by fractal analysis, through the estimation of Hurst index, for instance. However, as reported in literature [7], the LRD property itself is not sufficient for describing modern teletraffic, because such teletraffic exhibits not only LRD but also high bit rate variability (burstiness), when fractal analysis fall short in fully describing such process. Long-range dependency is only one feature of a "fractal" behavior describing mainly low frequency content (or the global trend) of the signal variability-see, for instance, R/S plot in Figure 2: Hurst index, as a descriptor of fractal nature, is obtained as a slope of linear regression line, although the slope locally varies with observed scale. Conversely, multifractal approach is capable to perform both local and global features of the process under investigation, being more appropriate for analysis of different complex processes, including compressed video.

We evaluated the LRD property of compressed video by observing Hurst indices, which are estimated for long video traces publicly available at [14]. The multifractal analysis was performed by histogram method. This method exploits coarse Hölder exponent, enabling sharp distinction between fine details in the MF spectrum permitting the selection and extraction of particular singularities, by applying an inverse MF analysis [32, 33]. Some other methods for estimating multifractal parameters, known from literature and publicly available, are compared with our method. Although global results are quite similar, irrespective of the method, when using our method the MF spectrum retains high frequency components permitting fine distinction between different processes. Moreover, our method enables an inverse MF analysis, meaning that from once derived MF spectrum we may recognize and extract video frames having particular value of a pair $(\alpha, f(\alpha))$.

The results performed by analyzing H.264/AVC video traces were compared to those obtained for the same sequences compressed by other coding standards, such as H.261, H.263, and MPEG-4, Version 2, of different quality. It was shown that for low quantization scale $q_{p}$ (or low compression ratio) MF spectrum corresponds to multiplicative process and exhibits mainly LRD behavior. Higher values of $q_{p}$ produce broader spectra indicating the higher multifractal 


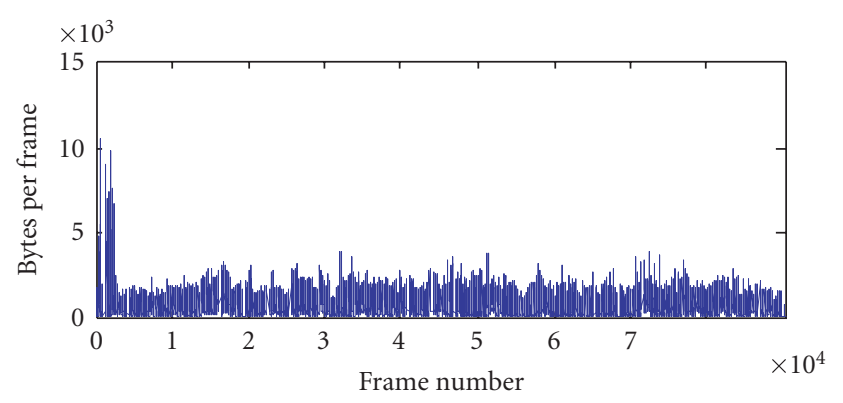

(a)

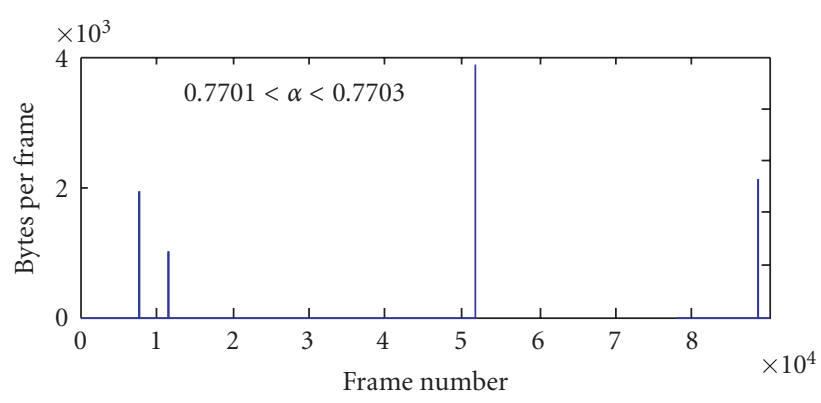

(b)

FIGURE 11: Illustration of inverse multifractal analysis: (a) input video trace "Starship Troopers" H.264/AVC, $q_{p}=10$; (b) extracted frames having particular values of $\alpha: 0.7710<\alpha<0.7703$.

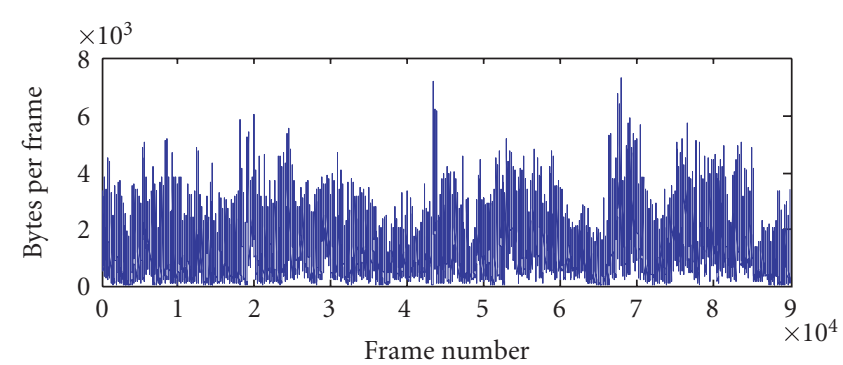

(a)

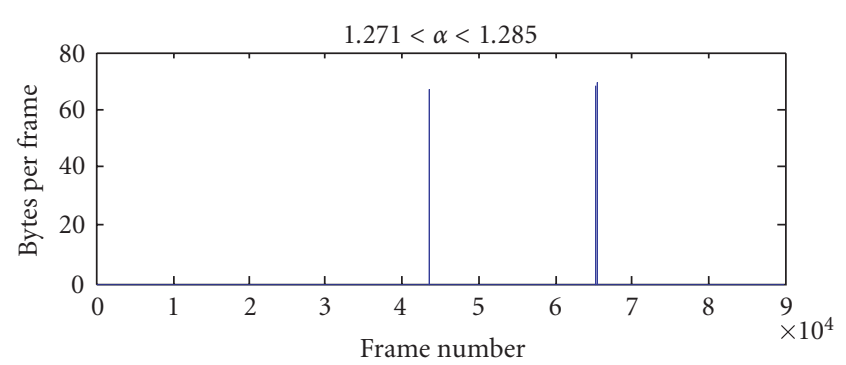

(b)

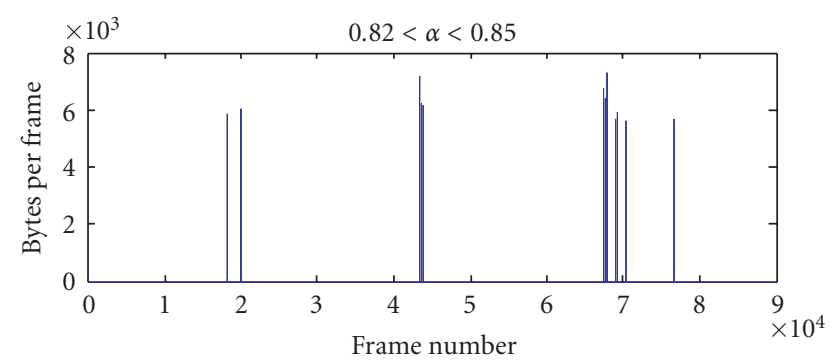

(c)

FIGURE 12: Illustration of inverse multifractal analysis: (a) input video trace "Starship Troopers," MPEG-4 V2, $q_{p}=10$, (b) extracted frames having particular values of $\alpha: 1.271<\alpha<1.285$; extremely rare events—very short frames, (c) extracted frames having particular values of $\alpha: 0.82<\alpha<0.85$; rare events-largest singular frames in a whole video.

nature. Furthermore, when $q_{p}$ increases, the spectra become more asymmetrical-going to higher $\alpha$ (indicating to patterns with higher fractality), having more local maxima, and more local singularities (indicating to additive processes). In addition, when $q_{p}$ increases the maximum of MF spectrum $f(\alpha)$, corresponding to the fractal dimension, migrates rightwise (to higher $\alpha$ ), while the value of $f(\alpha)_{\max }$ becomes lower. Such features indicate that higher $q_{p}$ increase local fractal behavior of most frequently events but the number of these events decreases. The statistics of the compressed video definitely are related to the rate of compression.

More detailed analysis of video traces shows that the main influence on the whole MF spectrum is that of the B frames, while I frames produces least influence. This conclusion is quite reasonable having in mind the compression mechanism: I frames are compressed exploiting spatial redundancy between pixels within the same frame, having thus the smallest compression rate and the smallest variability in size versus quantization scale. Conversely, $\mathrm{P}$ and $\mathrm{B}$ frames exploit mainly temporal redundancy being more compressed and more variable in size, that is, having stronger burstiness. As compression ratio increases, $\mathrm{P}$ and $\mathrm{B}$ spectra become more broader and more irregular indicating to higher multifractal nature of these traces.

From the results derived in this paper one can conclude that compressed digital videos, irrespective of the particular compression technique, exhibit inherent fractal property (or isolated fractal behavior), without any interaction with 
network or some other sources of variability. Moreover, such videos have high bit rate variability (burstiness), particularly at higher compression ratios. Since modern multimedia traffic consists mainly of compressed video, by analyzing such a video better understanding of modern teletraffic will be possible. Certainly, when sending compressed video over real network, traffic conditions influenced the signal and may change its Hurst index, both increasing or decreasing it, depending on particular case. For instance, when using neural network scheduling in packet switching node [15], outgoing traffic tends to be less fractal than the incoming one-the Hurst index decreases approaching to 0.5 (random walk process) $[23,24]$. The influence of external sources of variability to fractal/multifractal nature of compressed video will be our focus for further research.

\section{REFERENCES}

[1] K. Rao, Z. Bojkovic, and D. Milovanovic, Multimedia Communication Systems: Techniques, Standards, and Networks, Prentice-Hall, Englewood Cliffs, NJ, USA, 2002.

[2] Y. Wang, J. Osterman, and Y. Q. Zhang, Video Processing and Communications, Prentice-Hall, Englewood Cliffs, NJ, USA, 2002.

[3] R. Schäfer, T. Wiegand, and H. Schwarz, "The emerging H.264/ AVC standard," EBU Technical Review, no. 293, 2003.

[4] ITU-T, H.263 Recommendation, ITU-T, Geneva, Switzerland, 2000.

[5] Draft ITU-T Rec. H.264, ISO/IEC 14496-10, 2002 E.

[6] M. Garrett, Contributions toward real-time services on packet switched networks, Ph.D. thesis, Columbia University, New York, NY, USA, 1993.

[7] R. Riedi and J. L. Vehel, "Multifractal properties of TCP traffic: a numerical study," INRIA Research Report 3129, INRIA, Rocquencourt, Le Chesnay Cedex, France, 1997, http:// www.stat.rice.edu/\%7Eriedi/cv_publications.html.

[8] F. Fitzek and M. Reisslein, "MPEG-4 and H.263 video traces for network performance evaluation," TKN Technical Report TKN-00-06, Technical University Berlin, Berlin, Germany, 2000.

[9] M. Reisslein, J. Lassetter, S. Ratnam, O. Lotfallah, F. Fitzek, and S. Panchanathan, "Traffic and quality characterization of scalable encoded video: a large-scale trace-based study, part 1: overview and definitions," Tech. Rep., Telecommunications Research Center, Department of Electrical Engineering, Arizona State University, Tempe, Ariz, USA, 2002. http://peach.eas.asu.edu/index.html.

[10] M. Reisslein, J. Lassetter, S. Ratnam, O. Lotfallah, F. Fitzek, and S. Panchanathan, "Traffic and quality characterization of scalable encoded video: a large-scale trace-based study, part 2: statistical analysis of single-layer encoded video," Tech. Rep., Telecommunications Research Center, Department of Electrical Engineering, Arizona State University, Tempe, Ariz, USA, 2002. http://www.eas.asu.edu/trace.

[11] M. Reisslein, J. Lassetter, S. Ratnam, O. Lotfallah, F. Fitzek, and S. Panchanathan, "Traffic and quality characterization of scalable encoded video: a large-scale trace-based study, part 3: statistical analysis of temporal scalable encoded video," Tech. Rep., Telecommunications Research Center, Department of Electrical Engineering, Arizona State University, Tempe, Ariz, USA, 2002. http://www.eas.asu.edu/trace.
[12] F. Fitzek, M. Zorzi, P. Seeling, and M. Reisslein, "Video and audio trace files of pre-encoded video content for network performance measurements," Tech. Rep., Telecommunications Research Center, Department of Electrical Engineering, Arizona State University, Tempe, Ariz, USA, 2003. http://www.eas.asu.edu/trace.

[13] M. Krishna, V. Gadre, and U. Desai, Multifractal Based Network Traffic Modeling, Kluwer Academic Press, Boston, Mass, USA, 2003.

[14] www.acticom.de.

[15] I. Reljin, "Neural network based cell scheduling in ATM node," IEEE Communications Letters, vol. 2, no. 3, pp. 78-80, 1998.

[16] I. Reljin and B. Reljin, "Neurocomputing in teletraffic: multifractal spectrum approximation," in Proceedings of the 5th Seminar on Neural Network Applications in Electrical Engineering (NEUREL '00), pp. 24-31, Belgrade, Yugoslavia, September 2000 .

[17] B. Reljin and I. Reljin, "Multimedia: the impact on the teletraffic," in Book 2, N. Mastorakis, Ed., pp. 366-373, World Scientific and Engineering Society Press, Clearance Center, Danvers, Mass, USA, 2000.

[18] I. Reljin and B. Reljin, "Statistical and multifractal characteristics of H.263 compressed video streams," in Proceedings of 20th Symp. on New Technologies in Post and Telecomm. Traffic, pp. 193-205, Faculty of Traffic Eng., Belgrade, Yugoslavia, December 2002.

[19] I. Reljin and B. Reljin, "Fractal and multifractal analyses of compressed video sequences," Facta Universitatis (NIS) Series: Electronics and Energetics, vol. 16, no. 3, pp. 401-414, 2003.

[20] M. Taqqu, V. Teverovsky, and W. Willinger, "Estimators for long-range dependence: an empirical study," Fractals, vol. 3, no. 4, pp. 785-788, 1995.

[21] M. Roughan, D. Veitch, and P. Abry, "On-line estimation of the parameters of long-range dependence," in Proceedings of IEEE Global Telecommunications Conference (GLOBECOM '98), vol. 6, pp. 3716-3721, Sydney, NSW, Australia, November 1998.

[22] I. Beran, Statistics for Long-Memory Processes, Chapman \& Hall, New York, NY, USA, 1994.

[23] I. Reljin, A neural network control of ATM multiplexer, Ph.D. thesis, Faculty of Electrical Engineering, University of Belgrade, Belgrade, Yugoslavia, 1998.

[24] B. Reljin and I. Reljin, "Neural networks in teletraffic control: pro et contra?" in Proceedings of the 4th International Conference on Telecommunications in Modern Satellite, Cable and Broadcasting Services (TELSIKS '99), vol. 2, pp. 518-527, Niš, Yugoslavia, October 1999.

[25] B. Mandelbrot, The Fractal Geometry of Nature, W. H. Freeman, New York, NY, USA, 1983.

[26] H. Peitgen, H. Jurgens, and D. Saupe, Chaos and Fractals, Springer, New York, NY, USA, 1992.

[27] M. Turner, J. Blackledge, and P. Andrews, Fractal Geometry in Digital Imaging, Academic Press, New York, NY, USA, 1998.

[28] C. Evertsz and B. Mandelbrot, "Multifractal measures. Appendix B," in Chaos and Fractals, H. Peitgen, H. Jurgens, and D. Saupe, Eds., pp. 849-881, Springer, New York, NY, USA, 1992.

[29] P. Iannaccone and M. Khokha, Eds., Fractal Geometry in Biological Systems, CRC Press, Boca Raton, Fla, USA, 1996.

[30] J. L. Véhel and P. Mignot, "Multifractal segmentation of images," Fractals, vol. 2, no. 3, pp. 371-377, 1994. 
[31] J. L. Véhel, "Introduction to the multufractal analysis of images,” Tech. Rep., INRIA, Rocquencourt, Le Chesnay Cedex, France, 1996.

[32] I. Reljin and B. Reljin, "Fractal geometry and multifractals in analyzing and processing medical data and images," Archive of Oncology, vol. 10, no. 4, pp. 283-293, 2002.

[33] T. Stojic, I. Reljin, and B. Reljin, "Adaptation of multifractal analysis to segmentation of microcalcifications in digital mammograms," Physica A: Statistical Mechanics and its Applications, vol. 367, pp. 494-508, 2006.

[34] A. Chhabra and R. Jensen, "Direct determination of the $\mathrm{f}(\alpha)$ singularity spectrum," Physical Review Letters, vol. 62, no. 12, pp. 1327-1330, 1989.

[35] B. Gammel, MATPACK Library Release 1.4, http://users.physik.tu-muenchen.de/gammel/matpack/html/LibDoc/Tools/install.html.

[36] FracLab v1.1, 2003, http://www.irccyn.ec-nantes.fr/hebergement/FracLab.

Irini Reljin received the Diploma degree in electrical engineering in 1977, and the M.S. and Ph.D. degrees in electrical engineering in 1986 and 1998, all from the Faculty of Electrical Engineering (FEE), University of Belgrade, Serbia and Montenegro (former Yugoslavia). Since 1983 she is with the ICT College in Belgrade, working as a College Professor. Since 2001 she joined the FEE, University of Belgrade, as an Assistant Pro-

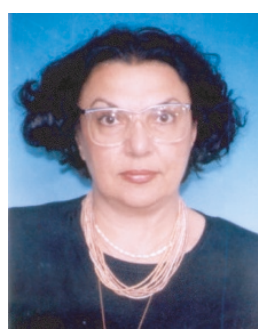
fessor, teaching the mulimedia and video technologies at undergraduate studies, as well as neural networks applications at graduate studies. She has published over 20 journal papers and over 150 conference presentations, as well as several book chapters, and has given a number of invited lectures on different aspects of communications, signal and image processing, fractal and multifractal analyses, and content-based indexing and retrieval. She has participated in a number of scientific and research projects in the areas of telecommunications, multimedia, and telemedicine, and currently she participated in COST Action 292 "Semantic Multimodal Analysis of Digital Media.” Her research interests are in video and multimedia analyses, and digital image processing, neural networks, statistical signal analysis, fractal and multifractal analyses. She is a Member of the IEEE, SMPTE, BSUAE, Gender Team, as well as several national societies.

Andreja Samčović received the Diploma and the M.S. degrees in electrical engineering in 1989 and 1995, respectively, from the Faculty of Electrical Engineering, University of Belgrade, Serbia and Montenegro (former Yugoslavia), and the Ph.D. degree in 2005 from the Faculty of Traffic and Transport Engineering, University of Belgrade. He joined the Faculty of Traffic and Transport Engineering, University of Bel-

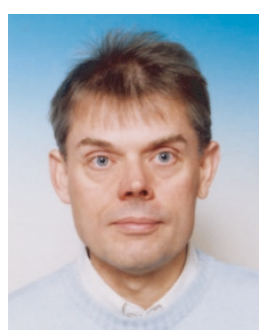
grade, from 1991, working in the areas of electrical engineering, communications, and image coding. He spent 9 months in $1991 / 1992$ as a scholar of the OEAD at the TU Vienna, Austria, and 6 months in 1999/2000 at the Friedrich-Alexander University Erlangen-Nuremberg, in Erlangen, Germany, as a scholar of DAAD. In 2003, he served as a lecturer at the UniAdrion summer school in Ammoudia-Preveza, Greece. In 2003-2004, he participated at the research project with the Virtual University of the Adriatic-Ionian basin, as well as in 2004-2005 at the bilateral project with Technical University of Kosice, Slovakia. He has published ten journal papers and over 60 conference presentations, on different aspects of image coding and communications, as well as one monograph. He is a Member of the BSUAE, and several national societies.

Branimir Reljin received the Diploma degree in electrical engineering in 1969, and the M.S. and Ph.D. degrees in electrical engineering in 1977 and 1983, all from the Faculty of Electrical Engineering (FEE) University of Belgrade, Serbia and Montenegro (former Yugoslavia). He joined the FEE, University of Belgrade from 1974, passing all teaching positions, and since 1995 he has been a Professor of electrical

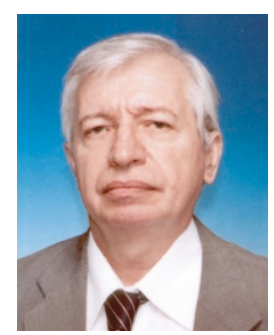
engineering, teaching several courses in graduate and undergraduate studies: circuit theory, digital image processing, medical informatics, telemedicine, artificial neural networks, digital signal processing, and network synthesis. He has published over 60 journal papers and over 250 conference presentations, four books and several book chapters, and has given a number of invited lectures, on different aspects of circuit theory, neural networks, medical image processing, fractal and multifractal analyses, and content-based indexing and retrieval. He has been a Project Leader in several scientific and research projects in the areas of neural networks, medical imaging and telemedicine, and signal acquisition and processing, and currently he is a Coordinator of Working Group 5 in COST Action 292 "Semantic Multimodal Analysis of Digital Media." He is a Member of the IEEE, BSUAE, and several national societies. 